\title{
MULTIVIEW DEPTH CODING BASED ON COMBINED COLOR/DEPTH SEGMENTATION
}

\author{
J. Ruiz-Hidalgo ${ }^{\mathrm{a}}$, J.R. Morros ${ }^{\mathrm{a}}$, P. Aflaki B. ${ }^{\mathrm{b}}$, F. Calderero ${ }^{\mathrm{c}}$, F. Marqués ${ }^{\mathrm{a}}$ \\ ${ }^{a}$ Department of Signal Theory and Communications \\ Universitat Politècnica de Catalunya \\ Jordi Girona 1-3, 08034, Barcelona, Spain \\ Fax: +34934016447, Phone: +34934016440 \\ Email: \{j.ruiz, ramon.morros, ferran.marques\}@upc.edu \\ ${ }^{b}$ Tampere University of Technology, Tampere, Finland \\ Email: payman.aflaki@tut.fi \\ ${ }^{c}$ Universitat Pompeu Fabra, Tànger 122, 08018, Barcelona, Spain \\ Email: felipe.calderero@upf.edu
}

\begin{abstract}
In this paper a new coding method for multiview depth video is presented. Considering the smooth structure and sharp edges of depth maps, a segmentation based approach is proposed. This allows further preserving the depth contours thus introducing fewer artifacts in the depth perception of the video. To reduce the cost associated with partition coding, an estimation of the depth partition is built using the decoded color view segmentation. This estimation is refined by sending some complementary information about the relevant differences between color and depth partitions. For coding the depth content of each region, a decomposition into orthogonal basis is used in this paper although similar decompositions may be also employed. Experimental results show that the proposed segmentation based depth coding method outperforms H.264/AVC and H.264/MVC by more than $2 \mathrm{~dB}$ at similar bitrates.
\end{abstract}

Keywords: Depth map, virtual view, multiview video coding, 3DTV 


\section{Introduction}

It is believed that $3 \mathrm{D}$ is the next major revolution in the history of TV. Both at professional and consumer electronics exhibitions, companies are eager to show their new 3D applications. In these applications, the 3D effect is obtained by recording the scene from multiple viewpoints, which is referred as Multi-View Video (MVV) [1]. MVV information is often completed with sample depth information (depth maps) for each view, thus conforming the Multi-view Video + Depth (MVD) format (Fig. 1). The introduction of this MVD format allows the incorporation of new functionalities such as Free View Point Video [2] in current 3DTV applications. MVD representations result in a vast amount of data to be stored or transmitted. In most situations, there is a need to compress these data efficiently without sacrificing visual quality significantly. Previous works presented various solutions for MVV coding (MVC) [3], mostly based on H.264 with combined temporal and inter-view prediction. Also, approaches for depth map coding in MVD have been proposed $[4,5,6]$.

In 3D applications, depth maps are used for rendering new images (virtual color views) but not to be directly viewed by the end user. Thus, the goal when coding depth maps is to maximize the perceived visual quality of the rendered virtual color views instead of the visual quality of the depth maps themselves [4]. Traditional image compression methods have been designed to provide maximum perceived visual quality, so using these methods to compress depth maps may result in suboptimal performance. The rendering error depends on the quality of the depth map coding and the quality of the 


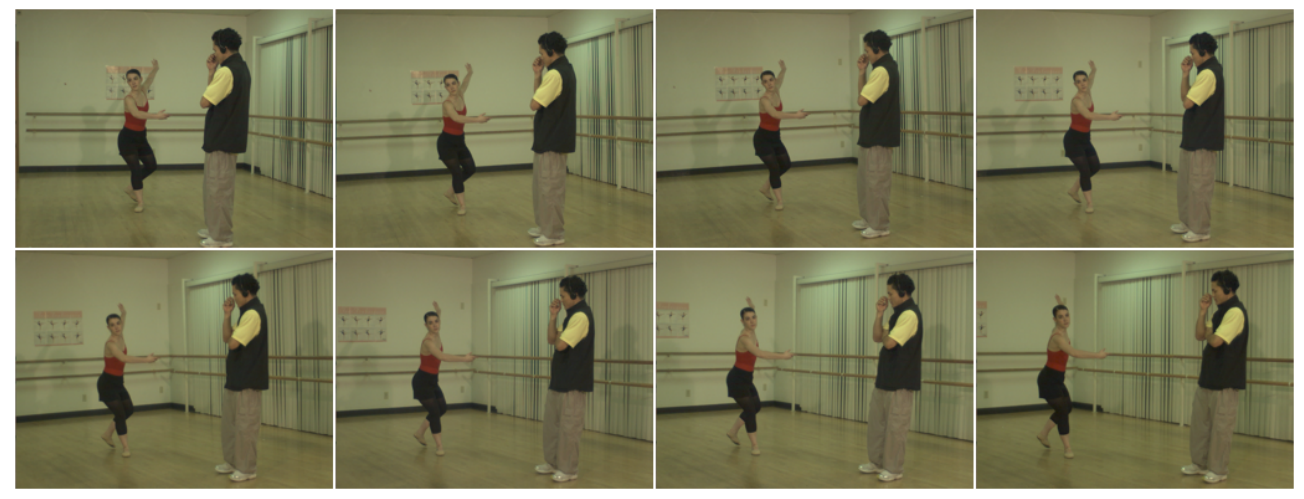

(a)

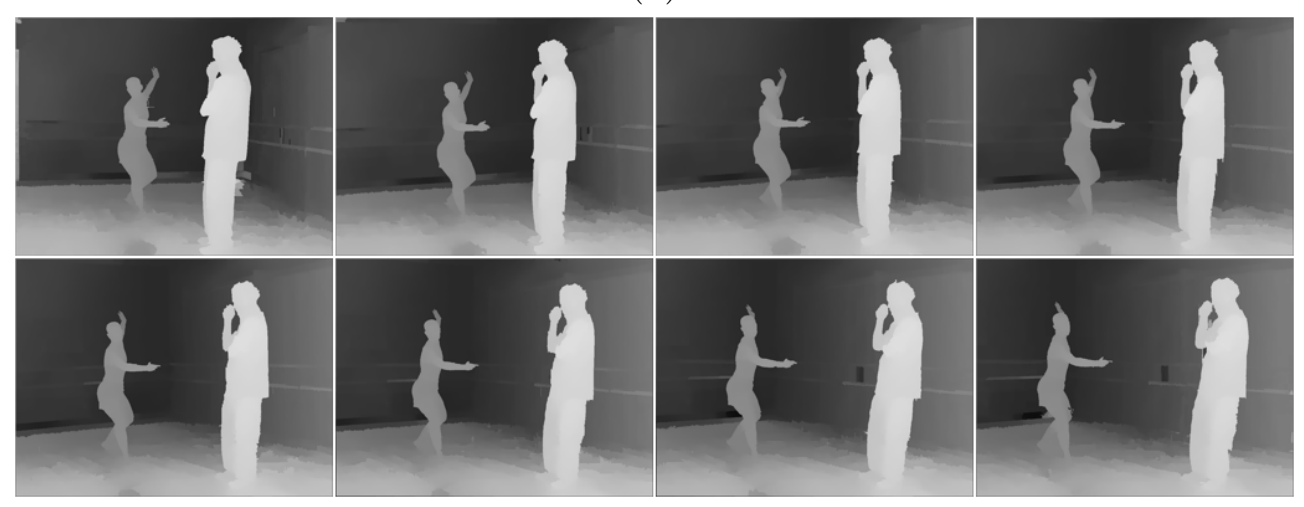

(b)

Figure 1: Multi-view video + Depth (MVD) ballet sequence composed of 8 camera views. (a) Color view for all cameras at frame $t=0$. (b) Corresponding depth information at the same time instant.

coding of the original color view used as a reference, but also on the structure of the depth map. For example: errors in the depth map close to an intensity edge can result in severe rendering artifacts, while errors on a smooth area may have negligible influence on the final quality.

These ideas are exploited, for instance, in [4], where the authors segment the depth map using a quadtree decomposition and then piecewise- 
linear functions are used to represent the depth information in each resulting block. Even though their experimental results show coding gains comparable to AVC with no temporal prediction (using only I frames) and MVC, costly partition information for the quadtree must be sent which results in a reduction of the performance. Another approach exploiting the previous ideas is presented in [7]. It relies on a shape-adaptive discrete wavelet transform (SA-DWT). The contours of the depth maps are extracted using a Canny edge detector and encoded using a differential Freeman chain-code. SA-DWT greatly reduces the data entropy of the resulting coefficients and the experimental results show significant coding gains with respect to traditional techniques based on DWT. However, a formal comparisons against the AVC standard is not provided. In [5], the authors proposed a combined representation, called layered depth image (LDI), for color views and depth in MVD sequences. As the LDI representation aims to reduce the redundant information between camera views, it is very useful for coding purposes. However, the number of layers for each element in the representation must be encoded lossless or near-lossless which limits its coding efficiency. Also, the LDI representation is more suited to scenarios with cameras close to each other.

Our proposal is inspired on segmentation-based coding systems (SBCS) [8]. These systems partition images (in this case, depth maps) into a set of arbitrarily shaped homogeneous regions. The separate coding of each region allows reducing the coding artifacts due to sharp edges and, hence, it is very appropriate to encode depth maps. As the need to send the partition information (shape of the regions) to the decoder is one of the major efficiency 
drawbacks of SBCS, we present a novel solution that greatly reduces the cost associated to depth partition coding using the fact that the depth partition can be estimated using the decoded image color partition. Experimental results in Section 3 will show that the proposed method outperforms AVC and MVC when encoding MVD sequences.

This paper is organized as follows. Section 2 describes the approach proposed to encode depth maps in multiview sequences using a segmentation based technique. Section 3 presents the experimental results comparing our method with AVC and MVC. Finally, Section 4 provides some conclusions and future extensions of this work.

\section{Segmentation based depth encoding approach}

Our proposal is based on segmenting the depth map into homogeneous regions of arbitrary shape and then coding the contents of these regions using texture coding techniques [8]. For simplicity, in the sequel we will use the word texture to refer to the depth information inside the regions. As can be seen in Fig. 1, depth maps can be considered approximately piecewise planar, with highly homogeneous regions separated by strong contours. The resulting regions can be efficiently encoded using low order methods, like piecewise linear functions in the case of [4] or a decomposition into orthogonal bases in our approach.

Having uniform regions will allow coding the depth values (texture) inside the region with only a few coefficients. However, as the receiver does not know the shape of the resulting regions, the partition must be encoded and transmitted. To avoid the high cost associated to coding the resulting 
partitions (region shape), instead of directly segmenting the depth map (as in other approaches, like [4]), the novel idea we are proposing is to approximate the depth partition from the decoded color partition, and to send only the information about the relevant difference between both partitions. As it will be shown, this will result in a very efficient coding of the depth partition.

In this process we make two assumptions: a) the color views have been previously coded and sent to the receiver (decoded color views), and b) we can always construct a color partition (generated using only color information) such that it contains the important depth transitions. This is, a homogeneous region in the color partition will also result in a homogeneous region in the depth partition. This ensures that depth regions can be encoded efficiently.

Assumption b) is not straightforward and needs further reasoning: In most cases, strong transitions in the depth maps coincide with color transitions in the color views because objects at different depths usually have different color or illumination conditions. Figs. 2.a and 2.b show a comparison between partitions computed on the color and depth images, both with the same number of regions. The strong depth transitions, separating both persons from the background, are also present in the color partition.

By using very fine color partitions, that is, with a high enough number of regions, as in Fig. 2.c, it can be ensured that almost all relevant contours in the depth partition are also present in the color view partition.

There are some cases where b) does not hold. One is when objects in the foreground have the same color as the background. The other is when objects with uniform color but slowly changing depth (i.e. walls or floors) are present in the image. 


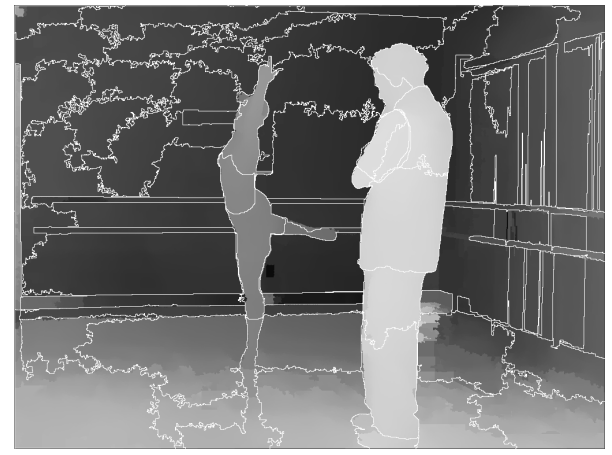

(a)

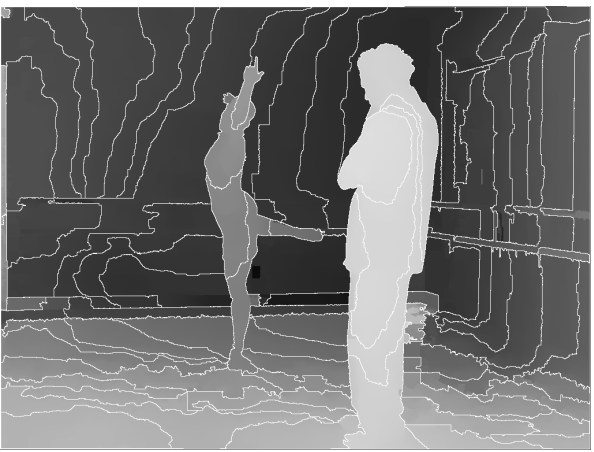

(b)

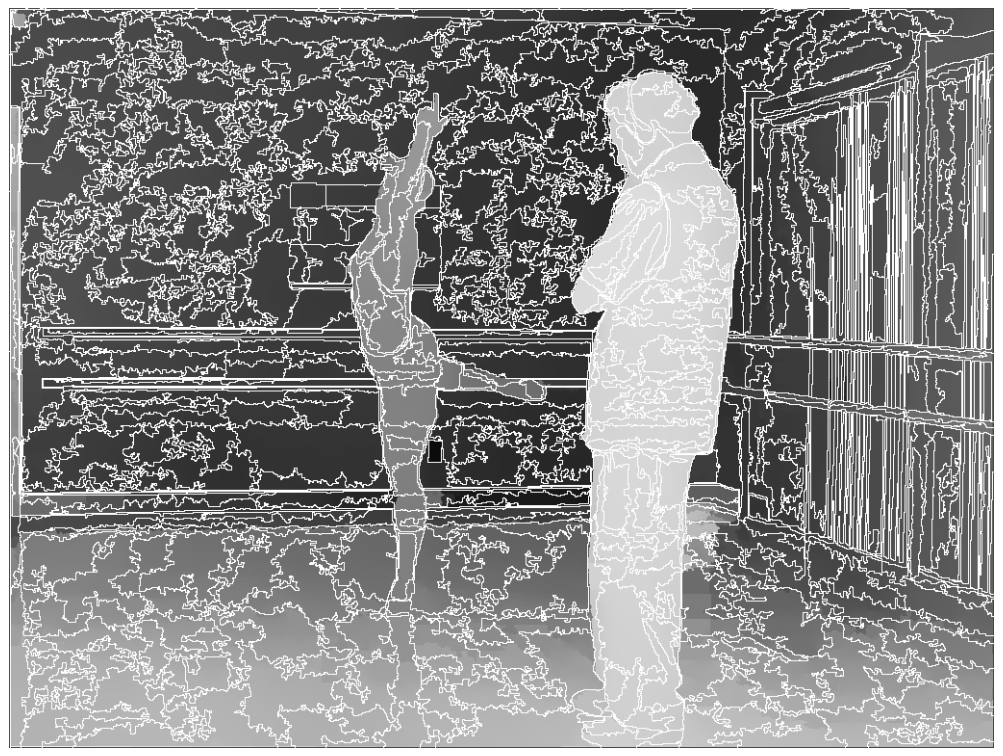

(c)

Figure 2: Comparison between a color partition and the corresponding depth partition at the same time instant. (a) Partition obtained using color homogeneity and (b) partition obtained using depth homogeneity for Ballet using 80 regions in each case. Finally, (c) is a color-based fine partition with 500 regions.

In the first case, our method will under-segment this zone and the coding will be less efficient because of a non-homogeneous region and the coded depth may have errors at the depth transitions, which can result in a quality 
loss at the rendered image. This effect can be seen, for instance, in Figs. 2.a and 2.b due to the similar color of the ballerina's hand and the wood handle at the bottom wall. The second case can be seen in Figs. 2.a and 2.b where, on the background (wall and floor) the shape of the color and depth regions differ. However, this does not result in quality loss because both partitions have smooth and low contrasted regions that can be efficiently coded.

Using the previous assumptions, a color view partition can be constructed both at the encoder and at the decoder that will contain all the significant contours in the depth partition (and probably, many more). We use the decoded color views also at the encoder side to ensure the same partitions are used at both sides. To further ensure region homogeneity when this partition is applied to the depth information, we construct it with a high number of regions (Fig. 2.c). This partition will be used to estimate the final depth partition. Looking at Fig. 2.c it is obvious that the color view partition is over-segmented with respect to the depth partition. This is caused by regions with similar depth but different color. Over-segmentation may result in coding inefficiency because of the large number of texture coefficients due to extra regions. By merging some of the regions of the decoded color view partition, a very good approximation to the optimal depth map partition can be constructed. That is, to get a better approximation of the depth map partition, we influence the color view segmentation process using information from the original depth map available only at the encoder. In this case, some side information must be sent to the decoder but its amount is small with respect to directly coding the depth map partition. 


\subsection{Color-depth based segmentation algorithm}

Our segmentation technique is based on a region-merging approach. Starting from an initial partition with a high number of regions (or even starting at the pixel level), the partition is constructed using an iterative process: at each iteration, the two more similar regions are merged. By stopping the merging process at a given level, we can obtain a partition with any desired number of regions.

The similarity measure is computed for each pair of neighboring regions. In our approach, region similarity is evaluated by comparing the models (for instance, region means) of the initial regions with the model of the resulting region after merging. The comparison criterion is the weighted euclidean distance between region models (WEDM [10]). For two regions $R_{1}$ and $R_{2}$, with areas $A_{1}$ and $A_{2}$ respectively, the WEDM cost, $O_{W E D M}$ is given by:

$$
O_{W E D M}\left(R_{1}, R_{2}\right)=A_{1}\left\|M_{R_{1}}-M_{R_{1} \cup R_{2}}\right\|_{2}+A_{2}\left\|M_{R_{2}}-M_{R_{1} \cup R_{2}}\right\|_{2}
$$

where the model $M_{R_{i}}$ for region $R_{i}$ is the vector formed by the average of all pixels values $I(p)$ with $p \in R_{i}$ :

$$
M_{R_{i}}=\frac{1}{A_{i}} \sum_{p \in R_{i}} I(p)
$$

The models can be computed on the YCbCr color space if segmentation is performed using color information or directly on the depth channel when depth information is used for depth segmentation In the first case, the cost will be referred as $O_{W E D M}^{C}$ and in the second case as $O_{W E D M}^{D}$. 


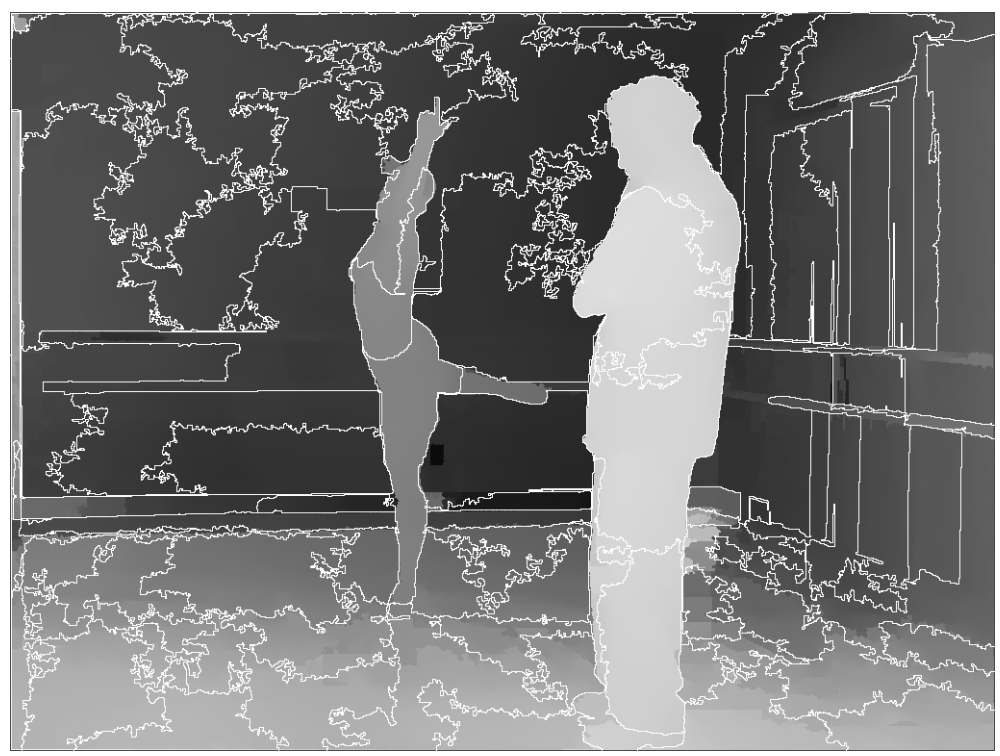

Figure 3: Example of final partition with 80 regions.

To create the final depth partition we use a process consisting of an estimation using a fine color partition and in a set of region mergings to better approximate the depth partition. This process can be described in three steps:

First step: A fine initial color view partition (initial partition) is built in the same way both at the encoder and the decoder (using the decoded color view) selecting a number of regions high enough to avoid under-segmentation (see Fig. 2.c). Section 2.1.1 details the algorithm used to determine the number of regions. This initial partition can be considered an estimation of the final depth partition and, since it can be constructed both at the encoder at the decoder, has no coding cost.

Second step: The final partition (Fig. 3) is obtained at the encoder side by using the aforementioned algorithm with WEDM cost in the depth 


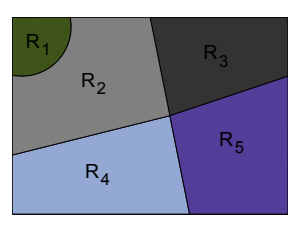

(a)

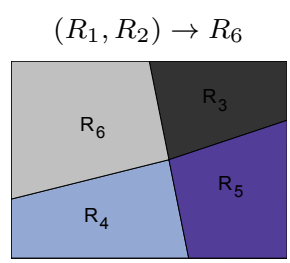

(b)

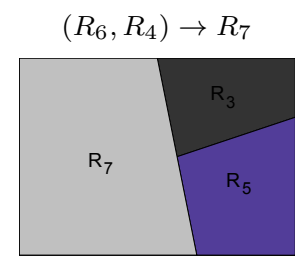

(c)

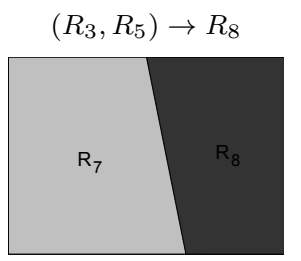

(d)

Figure 4: Example of creating a final partition of 2 regions. (a) Example of an initial partition computed from decoded color information using 5 regions. (b) First iteration after merging regions $R_{1}$ and $R_{2}$. (c) Second iteration after merging $R_{6}$ and $R_{4}$. (d) Final partition with 2 regions after all mergings have been performed.

information $\left(O_{W E D M}^{D}\right)$ starting from the initial partition constructed from the decoded color view. Using an initial partition constructed using only depth information would be optimal but the shape of this partition would need to be sent, at a high coding cost. The number of regions in the final partition is computed similarly to the initial partition (Section 2.1.1). The difference is that, for the initial partition, the criterion to select the number of regions was solely to avoid under-segmentation while in the case of the final partition, a number of regions suitable for coding purposes must be obtained.

Fig. 4 shows an example of the process followed by the encoder to compute a final partition of 2 regions. Starting from the initial partition represented in Fig. 4.a, the algorithm selects to merge the two regions with lowest $O_{W E D M}$ costs (eq. 1) computed in the original depth information $\left(O_{W E D M}^{D}\right)$. In the first iteration, the lowest cost correspond to regions $R_{1}$ and $R_{2}$ so they are merged in a new region $R_{6}$ (Fig. 4.b). The next step recomputes the $O_{W E D M}^{D}$ costs for the new region $R_{6}$ and selects again the lowest cost for the next 
merging. In the example, regions $R_{6}$ and $R_{4}$ are merged to $R_{7}$. The process is iterated until the final number of regions is found and the final partition is computed as in Fig. 4.d.

Third step: Even if the final partition is constructed from the initial partition, the decoder can not directly construct this partition because the depth information is not available. For that, the encoder re-computes the final partition but, this time, the decoded color information is used to calculate the $O_{W E D M}$ costs. At each iteration, the algorithm selects the two regions with lowest $O_{W E D M}^{C}$ cost to be merged but, if the two regions are not merged in the final partition, the merge is prevented and some side information is sent to inform the decoder so it is able to reproduce the final partition without the depth information. The side information is composed of a list of merging orders (called non-merging orders in this paper that inform the decoder if a given merge must be allowed or prevented. After a prevented merge, the next two more similar regions are examined until a suitable merging is obtained.

Fig. 5 illustrates this process. Starting from the same initial partition (Fig. 4.a) as in the second step, regions are merged based on their color similarity. For instance, in the first iteration, the lowest $O_{W E D M}^{C}$ cost corresponds between regions $R_{2}$ and $R_{3}$ (note that this first iteration is different than in the second step as the decoded color information is used to compute the cost). As regions $R_{2}$ and $R_{3}$ are not merged in the final partition computed in the previous step (Fig. 4.d) the merge is prevented (a 1 is stored in the non-merging orders list) and the next $O_{W E D M}^{C}$ cost is analyzed. In the example, the next lower costs correspond to regions $R_{4}$ and $R_{5}$. This merging 
is also prevented. All following costs are analyzed until one corresponding to two regions that have also been merged in the final partition. In the example, regions $R_{2}$ and $R_{4}$ are merged into region $R_{6}$ (Fig. 5.b) and a value of 0 is stored in the non-merging orders list. The process is iterated (Fig. 5.c) until the final number of regions (the same as the final partition) is achieved which results in a non-merging order of 110110110. The partition obtained in Fig. 5.d is identical to the final partition computed previously (Fig. 4.d). It is a reasonable approximation to a partition obtained using only depth information (without using the initial partition) while largely reducing the cost associated to partition coding.

The non-merging orders can be used in the decoder to replicate the encoder partition without any depth information. The cost associated to the non-merging orders is the only information needed for partition coding and its cost is very low compared to fully encoding the optimal depth partition. In our example, even though the worst possible scenario has been selected, only 9 bits are needed to encode the entire final partition. In real images, the associated cost $O_{W E D M}^{C}$ and $O_{W E D M}^{D}$ between two regions may be similar and the length of the non-merging orders would be reduced. Also, the probability of prevent mergings (denoted as 1 in the non-merging orders) is low (around 0.2 for our test sequences) thus allowing for a further compression using arithmetic coding.

The actual information sent to the decoder consists of: a) the number of regions for the initial and final partitions, b) the sequence of non-merging orders (the list of ones and zeros) and c) the texture coefficients for each resulting region. Any available encoding technique can be used to encode 


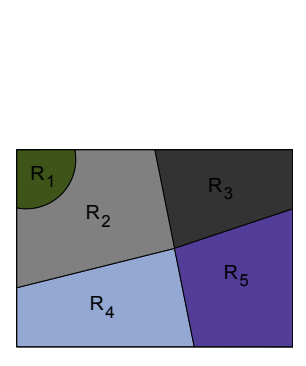

(a)

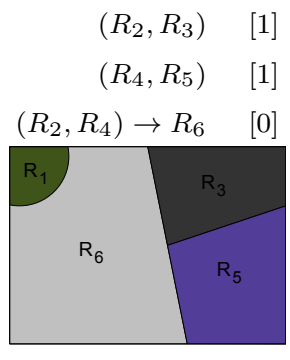

(b)

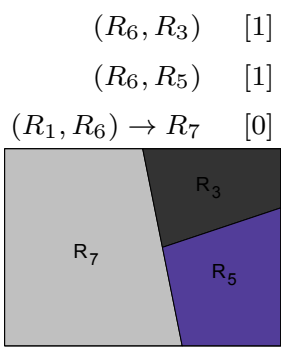

(c)

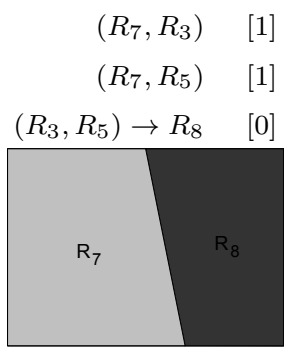

(d)

Figure 5: Example of computing the non-merging orders. Resulting non-merging order is 110110110.

the texture of each region. In this work, a decomposition into a set of basis functions (for example, polynomial) that are orthogonal to the shape of the regions (orthobasis) [11] has been used. The use of polynomial basis functions is a generalization of piece-wise linear functions and allow a better handling of the smooth variations inside the regions.

\subsubsection{Selection of the number of regions in initial and final partitions}

In the proposed algorithm, the initial and final partitions are constructed based on color uniformity (to ensure they can be replicated at the decoder), but must also fulfill an uniformity criterion when applied to the depth images. That is, we want to ensure that there are no strong depth transitions inside the regions, as this will result in a poorly encoded depth. Additionally, the partitions have a strong influence in the final coding cost.

In this work, the parameters used to control the initial and final partitions are their number of regions. Selecting an initial partition with a very large number of regions, ensures that there will not be under-segmentation but it may increase the number of non-merging orders to be coded. The same is 
true for the final partition: too few regions may result in non-homogeneous regions, while using too many regions the cost of the texture coefficients might result excessive.

In this work, the segmentation process is controlled applying measures commonly used for partition evaluation $[12,13]$. Unsupervised evaluation methods are the most suitable for this purpose because they are quantitative, objective and they require no reference images.

Most methods have some kind of intra-region uniformity criterion. Among all the available measures, the ones based on measuring contrast are very appropriate for our purpose. Texture coding techniques can efficiently represent regions with smooth texture variations with only a few coefficients, but perform worse if strong transitions (high contrast zones) are present on the area to code. Moreover, these techniques have low computational complexity.

Thus, selection of the number of regions is done using an intra-contrast measure $[13,14]$. Intra-region contrast measures the contrast inside the regions and has no penalty for over-segmentation. This criterion will be used to avoid under-segmentation in the initial partition and also to select the number of regions of the final partition.

Regarding this criterion, [13] note that it is not well adapted to textured images. Nevertheless, in this application this is not a problem because it will be evaluated on the depth image, which is not textured at all.

Let $c(s, t)=\frac{|f(s)-f(t)|}{L-1}$ be the contrast between two pixels $s$ and $t$, with $f$ representing the intensity and $L$ the maximum intensity. The intra-region contrast for region $R_{i}$ with area $A_{i}$ is: 


$$
I_{i}=\frac{1}{A_{i}} \sum_{s \in R_{i}} \max \left\{c(s, t), t \in W(s) \cap R_{i}\right\}
$$

where $W(s)$ is a neighborhood of pixel $s$.

We can define a global measure based on the intra-region contrast. The global intra-region contrast can be defined as:

$$
I C=1-\frac{1}{N} \sum_{i=1}^{N} I_{i}
$$

where $N$ is the number of regions in the partition. The global measure is normalized to one and higher values of the scores are associated with better partitions.

To select the desired number of regions in the initial partition, we evaluate the global intra-region contrast measure (eq. 4) on the depth image during the construction of the initial partition and proceed with the region merging process while the measure is above a given threshold $t_{F P}$. This threshold marks the point with the maximum acceptable under-segmentation and can be determined empirically.

The same criterion is used to determine the number of regions in the final partition, using a different threshold $t_{I P}$. This will ensure that the number of regions is the minimum that still fulfills the under-segmentation criterion.

\section{Experimental results}

Experimental results are provided for two sequences: Ballet and Breakdancers [15]. Each sequence includes eight synchronized cameras with both depth and color view information through time. The resolution of both color 
view and depth maps is $1024 \times 768$ pixels and the duration of the sequences is 100 frames for each camera at 15 frames per second.

To compare our proposed technique, depth maps are encoded using the standard AVC [16] and MVC [3] codecs. As our proposed method does not currently exploit any temporal correlation between depth maps, two different configurations for the AVC codec have been used. The first one (All I frames) does not include any temporal prediction and uses only I frames for the entire depth sequence. The second configuration (Optimized) uses a standard IPB GoP configuration of 12 frames (IBPBPBPBPBPB). The MVC codec is also configured using the same GoP length, with temporal and interview prediction.

Fig. 6 presents examples of the original and decoded depth maps using both Optimized $A V C$ and the proposed method at similar bitrates. It can be seen that the proposed method preserves better the contour information and avoids the blurring/blocking artifacts that appear in the AVC or MVC encoded images (Fig. 6.b) introducing only some small artifacts caused by under-segmentation errors (Fig. 6.c).

In Fig. 6, the PSNR between a single frame of the original depth and the Optimized AVC encoded depth is $32.4 \mathrm{~dB}$ while the same frame in the proposed method achieves $28.1 \mathrm{~dB}$ (both at a similar bitrate of 484.24 and $481.32 \mathrm{kpbs}$ for the entire MVD sequence respectively). However, depth maps are not intended to be viewed directly but employed to generate virtual views. For this reason, PSNR calculated on depth maps is not the best measure for applications involving virtual-view reconstruction. Errors at the border of depth discontinuities have a stronger contribution to reconstructed 


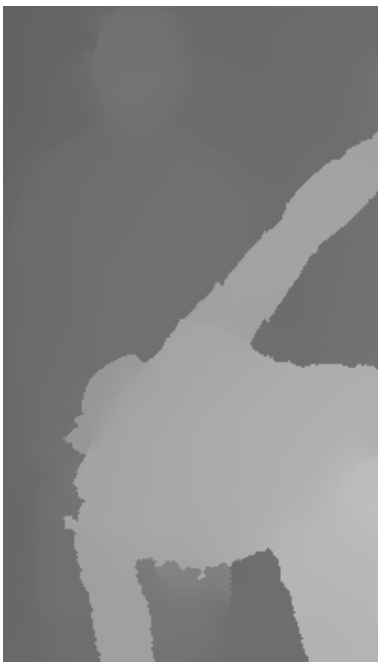

(a)

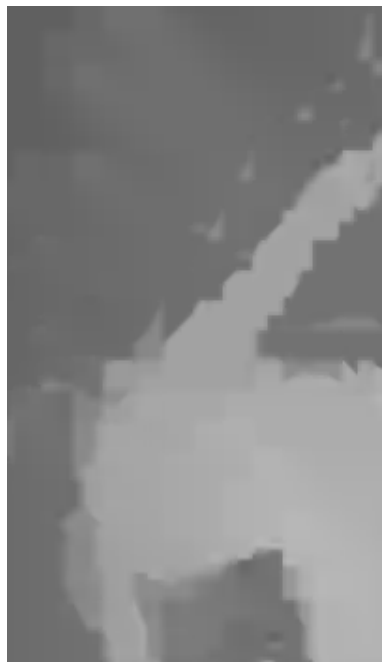

(b)

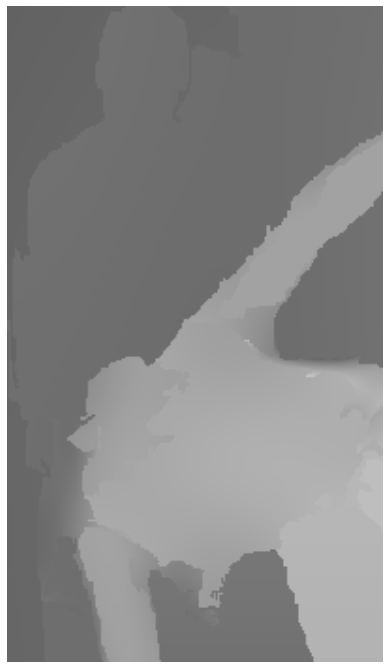

(c)

Figure 6: Direct quality comparisons between original and decoded depth maps at frame 15 of the Breakdancers sequence. (a) Original, (b) Optimized AVC with $32.4 \mathrm{~dB}$ at 484.24 kbps (c) Proposed method with $28.1 \mathrm{~dB}$ at 481.32 kbps.

color view distortions than errors in zones with smooth depth. Therefore, the methodology used in [4] will be applied to compute the PSNR between virtual color views instead of directly over the encoded and original depth maps. As it is shown in the sequel, the proposed method preserves more carefully the depth discontinuities, ensuring a better color view reconstruction even with worse results for PSNR measured in the depth maps. On the other side, H.264 encoders are highly optimized to achieve excellent Rate-Distortion performance when measuring directly the depth maps PSNR but may fail at preserving the contours of the objects.

Experimental results show a comparison between the proposed method, AVC and MVC. For the initial partition, $t_{F P}=0.990$ has been used as a threshold to determine the number of regions. In the case of the final 
partition, a value of $t_{I P}=0.975$ has been used. These two thresholds have been determined empirically by testing different images and have been used in all the results in the sequel. They result in approximately 500 regions for Ballet and Breakdancers in the initial partition and 75/80 regions in the final partition.

To encode the regions content, a second order orthobasis is employed and each coefficient is represented using 8 bits. At each frame, all coefficients for all regions are grouped and entropy coded using an adaptive arithmetic codec.

The color views are encoded using Optimized $A V C$ with quantization step $\mathrm{Q}=32$ and we assume they are available to the decoder. Using such a high $\mathrm{Q}$ is a worst case scenario for our method as it decreases the quality of the decoded color view partition. Fig. 7 shows the PSNR for virtual color views along the 8 reference cameras for both Ballet and Breakdancers sequences (obtained using the original and encoded depth maps). Nine virtual color views are placed between each two reference cameras to estimate how the final quality is affected by the distance from the reference cameras. As reported in [4], PSNR values of virtual color views are significantly higher (around 35dB) than PSNR values measured directly between original and decoded images. This is because both virtual views are generated using the same color view; only the original and decoded depth map is changed, resulting in more similar images and higher PSNR values.

Moreover, Fig. 7 shows a common trend of PSNRs measured in virtual views, as the virtual view is located closer to one of the reference cameras, the PSNR always increases even though the encoded depth map is the same 
for all those virtual views. Thus, forming a characteristic "U" shape. Virtual views in the middle of reference cameras need to make more use of the depth map information than virtual views closer to one of the reference cameras. Therefore, are more sensible to artifacts or errors in the decoded depth maps. However, virtual views close to the reference cameras do not use depth map information that much and the resulting virtual view quality is greater (thus, increasing the PSNR). In the limit, a virtual view at the same place of the reference camera will yield an infinite PSNR.

Table 1 shows the bitrates obtained encoding the depth maps with all methods shown in Fig 7 . The final bitrate is obtained by adding the bitrates of the eight views. In the proposed method, the bitrate is composed of the bits needed to allow the receiver building the final partition (list of differentially encoded non-merging orders, number of regions in the initial and final partitions) plus the bits needed to encode depth information for each region (texture coefficients).

Table 1: Proposed method, AVC and MVC comparison (similar PSNR).

\begin{tabular}{l|c|c|c|c} 
& \multicolumn{2}{|c|}{ Ballet } & \multicolumn{2}{c}{ Breakdancers } \\
& $\mathrm{kbps}$ & $\mathrm{dB}$ & $\mathrm{kbps}$ & $\mathrm{dB}$ \\
\hline Proposed (Texture coeffs) & $277.2(57.6 \%)$ & & 285.08 & \\
\hline Proposed (Non-merging orders) & $204.12(42.4 \%)$ & & 203.72 & \\
\hline Proposed (Total) & 481.32 & 37.39 & 488.8 & 38.37 \\
\hline AVC All I frames & 2346.66 & 36.94 & 1317.42 & 38.53 \\
\hline AVC Optimized & 813.51 & 37.02 & 648.03 & 38.07 \\
\hline MVC & 843.62 & 37.17 & 632.04 & 38.28
\end{tabular}



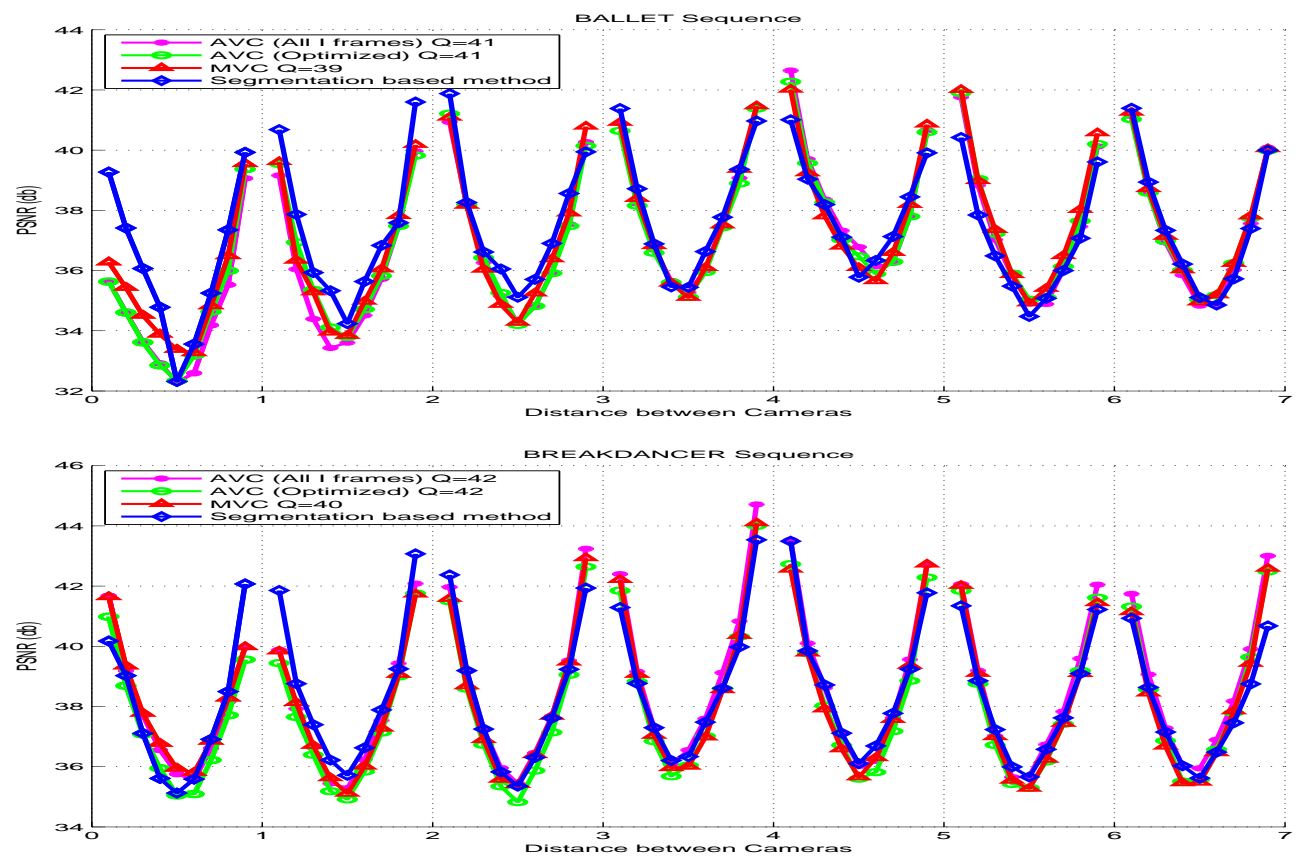

Figure 7: Comparison between the proposed method, AVC and MVC for sequences Ballet and Breakdancers at similar PSNR

As shown in Table 1, the total bitrate of our proposed method is much lower than the All I frames AVC configuration. Even comparing with the Optimized $A V C$ or the $M V C$ configurations, the resulting bitrate decreases around $43 \%$ (for Ballet) and 22\% (for Breakdancers) while keeping similar PSNR values. Also, mean PSNR values in dBs for virtual color views are shown in Table 1 for each coding configuration.

In Fig. 8 and Table 2 we can see an analogous comparison, this time for similar bitrates. In this case, the All I frames AVC configuration obtains a quality several dBs below the other two approaches and is not presented. Gains up to $2.1 \mathrm{~dB}$ and $1.9 \mathrm{~dB}$ are achieved for the Ballet and Breakdancers sequences respectively (1.4 dB and $0.8 \mathrm{~dB}$ in average). Experimental results 
show that the more efficient MVC codec does not even outperform the AVC codec proving that the higher R-D efficiency of the MVC does not directly translate into higher PSNR values of the virtual color views.
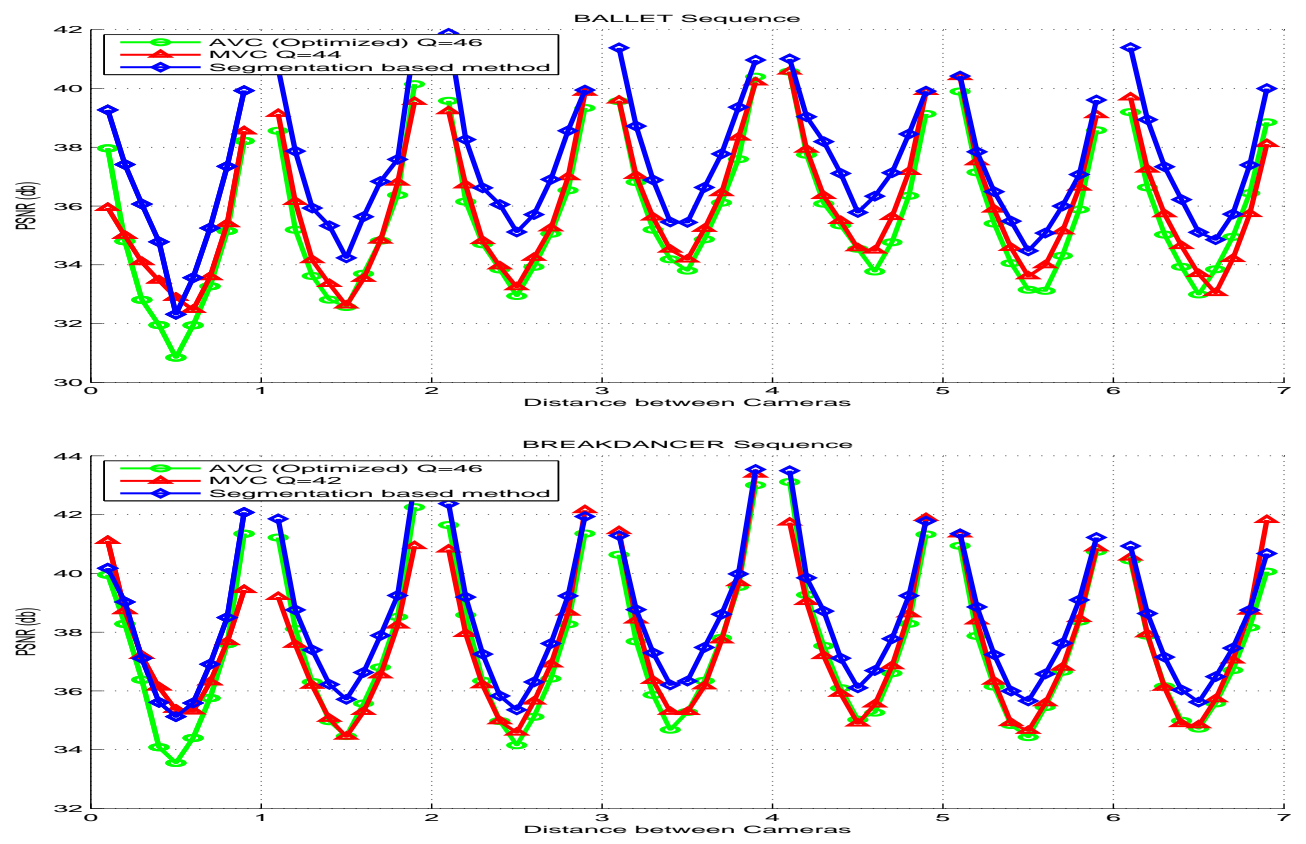

Figure 8: Comparison between the proposed method, Optimized $A V C$ and $M V C$ for sequences Ballet and Breakdancers at similar bitrate.

\section{Conclusion and future work}

In this paper, we have presented a method for depth map compression based on a novel approach. The main contribution of this paper is to exploit the redundancy between the color views and depth maps by using a combination of decoded color view and depth partitions. Color partitions are computed on the decoded views, which reduces the high cost commonly associated to partition coding. Depth partitions in the decoder are built 
Table 2: Proposed method, AVC and MVC comparison (similar bitrate).

\begin{tabular}{l|c|c|c|c} 
& \multicolumn{2}{|c|}{ Ballet } & \multicolumn{2}{c}{ Breakdancers } \\
& $\mathrm{kbps}$ & $\mathrm{dB}$ & $\mathrm{kbps}$ & $\mathrm{dB}$ \\
\hline Proposed (Texture coeffs) & $277.2(57.6 \%)$ & & 285.08 & \\
\hline Proposed (Non-merging orders) & $204.12(42.4 \%)$ & & 203.72 & \\
\hline Proposed (Total) & 481.32 & 37.39 & 488.8 & 38.37 \\
\hline AVC Optimized & 484.24 & 35.67 & 468.32 & 37.46 \\
\hline MVC & 485.68 & 35.99 & 495.64 & 37.59
\end{tabular}

combining a decoded color view segmentation along with some overhead complementary information from the original depth segmentation. Comparing the final results with AVC and MVC, we achieve gains of more than $2 \mathrm{~dB}$ for similar bitrates or reductions in bitrate around $40 \%$ for similar PSNR. While the proposed method assumes the availability of the color views in the decoder, we believe it is reasonable to expect so as depth maps are always associated to color views to provide functionalities of free view point video or 3DTV.

It is also important to note that the proposed method currently encodes depth maps separately for each frame and neither temporal nor inter-view prediction is performed so far. By exploiting these redundancies (both in the final partition and in the depth information coding steps) we expect to achieve even higher gains. Moreover, the use of a Binary Partition Tree (BPT) [9] to represent the region-merging sequence will allow us to use a rate-distortion approach to find an optimal segmentation, for instance by selecting the best $t_{I P}$ and $t_{F P}$ thresholds or the optimal number of regions 
in the initial and final partitions. Finally, alternative region-based texture coding methods, such as DCT or partial wavelets, will be studied in order to improve the depth information coding currently based on the orthobasis approach.

\section{References}

[1] H. Ozaktas, L. Onural, Three-Dimensional Television: Capture, Transmission, and Display, Springer, Heidelberg, 2007.

[2] A. Smolic, K. Müller, P. Merkle, C. Fehn, P. Kauff, P. Eisert, T. Wiegand, 3D video and free viewpoint video technologies, applications and MPEG standards, in: IEEE International Conference on Multimedia and Expo, Toronto, Canada, pp. 2161-2164.

[3] P. Merkle, A. Smolic, K. Müller, T. Wiegand, Efficient prediction structures for multiview video coding, IEEE Trans. on CSVT (2007) 14611473.

[4] P. Merkle, Y. Morvan, A. Smolic, D. Farin, K. Müller, P. H. N. de With, T. Wiegand, The effects of multiview depth video compression on multiview rendering, Image Commun. 24 (2009) 73-88.

[5] S.-U. Yoon, Y.-S. Ho, Multiple color and depth video coding using a hierarchical representation, IEEE Trans. on CSVT 17 (2007) 1450-1460.

[6] M. Maitre, Y. Shinagawa, M. Do, Wavelet-based joint estimation and encoding of depth-image-based representations for free-viewpoint rendering, IEEE Trans. on Image Processing 17 (2008) 946-957. 
[7] M. Maitre, M. N. Do, Shape-adaptive wavelet encoding of depth maps, in: Picture Coding Symposium, volume 27, Chicago, USA, pp. 473-476.

[8] L. Torres, M. Kunt, Video Coding: The second generation approach, Kluwer Academic Publishers, 1996.

[9] P. Salembier, L. Garrido, Binary partition tree as an efficient representation for image processing, segmentation and information retrieval, IEEE Trans. on IP 9 (2000) 561-576.

[10] V. Vilaplana, F. Marques, P. Salembier, Binary partition trees for object detection, IEEE Trans. on Image Processing 17 (2008) 1-16.

[11] M. Gilge, T. Engelhardt, R. Mehlan, Coding of arbitrarily shaped image segments based on a generalized orthogonal transform, Signal Processing: Image Communication 1 (1989) 153-180.

[12] H. Zhang, J. E. Fritts, S. A. Goldman, Image segmentation evaluation: A survey of unsupervised methods, Computer Vision and Image Understanding 110 (2008) $260-280$.

[13] S. Philipp-Foliguet, L. Guigues, Multi-scale criteria for the evaluation of image segmentation algorithms, Journal of Multimedia 3 (2008).

[14] J.-P. Cocquerez, S. Philipp, Analyse dimages: filtrage et segmentation, Masson, Paris, 1995.

[15] Interactive Visual Media Group of Microsoft Research, MSR 3D video download, http://research.microsoft.com/enus/um/people/sbkang/3dvideodownload/, 2005. 
[16] ITU-T, ISO/IEC JTC 1, Advanced video coding for generic audiovisual services, ITU-T Recommendation H.264 and ISO/IEC 14496-10 (MPEG4-AVC), Version 4: July 2005. 